\title{
Simultaneous Out-of-band Interference Rejection and Radiation Enhancement in an Electronic Product via an EBG Structure
}

\author{
Ruaro, Andrea; Thaysen, Jesper; Jakobsen, Kaj Bjarne
}

Published in:

Proceedings of the International Microwave Symposium (IMS 2014)

Link to article, DOI:

$10.1109 /$ mwsym.2014.6848352

Publication date:

2014

Document Version

Early version, also known as pre-print

Link back to DTU Orbit

Citation (APA):

Ruaro, A., Thaysen, J., \& Jakobsen, K. B. (2014). Simultaneous Out-of-band Interference Rejection and Radiation Enhancement in an Electronic Product via an EBG Structure. In Proceedings of the International Microwave Symposium (IMS 2014) IEEE. https://doi.org/10.1109/mwsym.2014.6848352

\section{General rights}

Copyright and moral rights for the publications made accessible in the public portal are retained by the authors and/or other copyright owners and it is a condition of accessing publications that users recognise and abide by the legal requirements associated with these rights.

- Users may download and print one copy of any publication from the public portal for the purpose of private study or research.

- You may not further distribute the material or use it for any profit-making activity or commercial gain

- You may freely distribute the URL identifying the publication in the public portal 


\title{
Simultaneous Out-of-band Interference Rejection and Radiation Enhancement in an Electronic Product via an EBG Structure
}

\author{
Andrea Ruaro*†, Jesper Thaysen*, and Kaj B. Jakobsen ${ }^{\dagger}$ \\ ${ }^{*}$ GN ReSound A/S, Lautrupbjerg 7, DK-2750 Ballerup, Denmark \\ ${ }^{\dagger}$ Department of Electrical Engineering, Technical University of Denmark, DK-2800 Lyngby, Denmark
}

\begin{abstract}
This work presents an application of a planar electromagnetic band gap (EBG) structure with a perspective product implementation in the back of the mind. The focus is on the integration of such structure under the constraint of space and system coexistence. It is discovered that it is possible to achieve simultaneously both the enhancement of the antenna radiation efficiency and the shrinking of its dimensions, while making the device more resilient to out-of-band electromagnetic interference (EMI). The patterning of the ground plane allows, in fact, to effectively suppress higher-order resonances (alternatively, parallel plate noise) and decrease the radiation efficiency of the structure forbidding higher-order modes to propagate and subsequently be diffracted by the ground plane.
\end{abstract}

Index Terms-Electromagnetic interference (EMI); electromagnetic compatibility (EMC); electromagnetic band gap (EBG) structures; small antennas; printed circuit boards (PCB)

\section{INTRODUCTION}

Electronic products such as handsets, mobile phones, wireless medical devices [1], etc..., are subjected to regulation concerning their electromagnetic compatibility (EMC) performance. Since their either main or accessory function is to communicate, they are substantially composed by at least a radio system comprising of a radio circuit and an antenna, and of a digital signal processor (DSP). In most cases, their dimensions and integration are constrained following the directions given by different industrial functions such as other engineers, mechanical designers, and marketing. For the electronics, this translates into a high level of integration, with all the functions confined on a small printed circuit board (PCB). Fig. 1 shows as an example all the space available in a given wireless medical device. The mutual proximity eases the jamming among sub-systems, in particular the analogue signals of the radio and the digital signals of the DSP can couple to antenna feed, power distribution network, high-speed interconnects, etc..., and ultimately be radiated as spurious emissions.

EBG structures, either planar or three-dimensional, have been proven able to filter EM noise from transmission lines and PCBs [2]-[4]. In fact, either the lines couple to the resonant structures of the EBG so that the energy of the propagating unwanted mode is reduced, or the EBG proves able to suppress the parallel plate resonance possibly occurring,

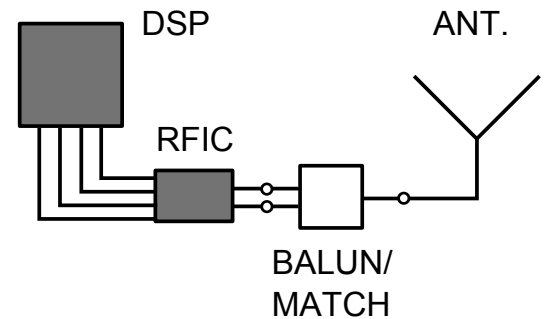

(a)

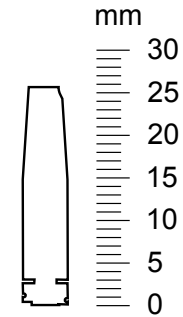

(b)
Fig. 1: Diagram (a) and PCB outline example (b), with related scale, of a compact-size electronic product having a wireless interface.

e.g., between the power and ground planes [5]. Alternatively, when the application demanded, defected ground structures (DGS) or metamaterial-inspired structures [6]-[8] have also been deployed with success to reduce interference, either from a full board or from scattered radiation sources confined on an area of the PCB. These signals are in general spread over a wide frequency spectrum.

On the other hand, EBG structures have been used in recent years to enhance radiation properties of antennas [9], [10]. Nevertheless, these works do not include considerations on their integration or performance versus EMI. A patch antenna is strongly dependent upon its ground plane structure, and therefore its radiation characteristics are notably influenced by it. The parameters that are mostly impacted are physical dimensions, directivity, bandwidth, and efficiency.

It has been left unanswered by literature studies if, choosing carefully the design of the EBG structure, the designer can simultaneously obtain an enhancement of the antenna radiation efficiency and bandwidth, while suppressing the noise coming from other transmission lines and the PCB itself. This work focuses on the design of an antenna with improved out-ofband spurious emissions performance, and brings the example of a device that has an alarming level of radiated power due to the power amplifier harmonics.

The article is organized as follows. Section II explains the set-up and the ground for its modelling. Section III presents and elaborates the results from FEM simulations. Finally, 


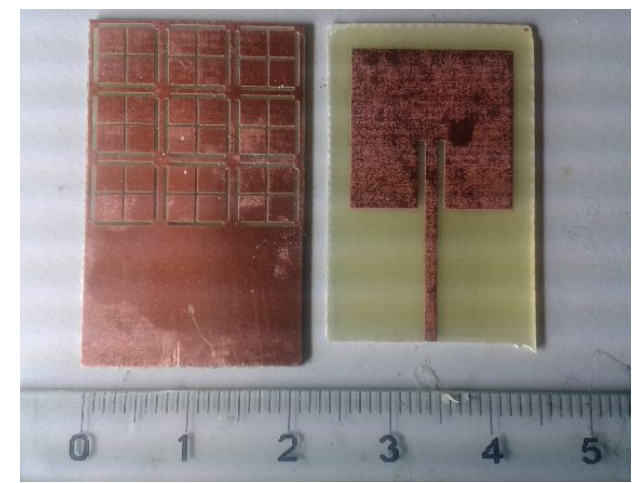

Fig. 2: Picture of the prototype antenna and its ground plane.

Section IV summarizes the findings.

\section{THEORY AND SET-UP}

A square patch antenna is chosen as the active element. Even though it can be objected that this configuration is not the most common one found in compact electronics devices, its advantages are many: a well-known radiation model; the amount literature on the interaction of plates with EBG structures; and its being compatible with product needs (cost, weight, mechanical integration, ....). Furthermore, the discussion can easily be extended without loss of generality to the parallel plate noise emitted from PCBs, due to the similar radiation mechanism, so to include resonances rooted into the geometry of ground and power planes, etc....

The modified antenna uses a ground plane on which is periodically etched a Jerusalem Cross pattern in a complementary fashion, as visible from the prototype picture in Fig. 2. This is a known planar implementation of an EBG structure, and allows a significant shrinking of the antenna dimensions. A simple model of EBG is used for design purposes [11], whereas tuning is left to parametric studies accomplished via software simulation [12]. Cost and space constraints, common to all mass produced products, dictates the use of a planar structure for the EBG implementation. The square patch antenna has a dimension of $16.3 \times 16.3 \mathrm{~mm}$ and operates in the $2.4 \mathrm{GHz}$ ISM band, it is fed via a recessed microstrip line, and it is implemented on a $0.8 \mathrm{~mm}$ thick FR4 substrate. The work uses as reference a traditional patch antenna with a small solid ground plane.

The peculiarity, compared to other studies in the sector, of making use of an antenna as a radiation source, instead of a simple metal plate, find its reason in the focus on unwanted signals, e.g., power amplifier (PA) harmonics, coupled at the input of a structure actually intended to radiate and therefore potentially more critical for the EMC performance.

A major drawback of the set-up is that is not possible to focus on the radiation pattern. In fact, the latter is markedly different at the center frequency between the two configuration and is therefore not eligible to represent a comparison term. Nevertheless, in small antennas the metric of most interest is the antenna efficiency - that can be similarly expressed

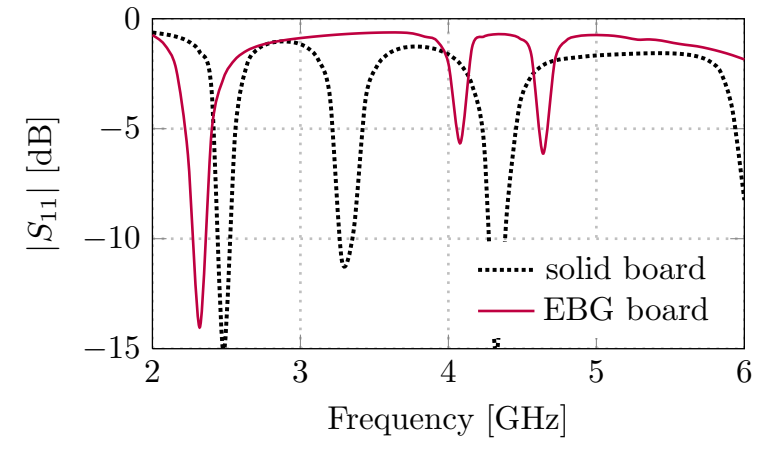

Fig. 3: Reflection coefficient for the two configurations.

through the total radiated power (TRP):

$$
T R P=P_{\text {cond }} \cdot \eta_{\text {rad }},
$$

where $P_{\text {cond }}$ is the conducted power of the radio and $\eta_{\text {rad }}$ the radiation efficiency of the antenna. The results are therefore compared in terms of the radiation efficiency of the structure. This is defined as the power radiated by an antenna over the power accepted at its terminals, therefore the result is independent from the matching condition. Even though TRP is not necessarily the most indicative metric with respect to EMC testing, since it doesn't take into account the directionality of the radiation, it is the most effective in the present context.

\section{Simulation Results}

Fig. 3 shows the reflection coefficient for the two configurations. It is clearly noticeable how the EBG structures affects the resonance frequencies of the patch. It can also be read a slight improvement in bandwidth.

Fig. 4 shows the comparison between the simulated efficiency of the two antennas versus the frequency. The radiation efficiency is satisfactory in the operating band for both geometries, but it is appears clearly that the antenna based on the EBG substrate is more performing. At a certain point in frequency, the two curves assume the same value and for the whole frequency range above the EBG structure radiates instead less effectively. This is desirable, since it is going to reduce the level of the spurious emissions above the crossing frequency, in a more or less marked fashion as compared to the reference design.

A patch supports higher-order resonances, that radiates according to its characteristic modes. In proximity of these frequencies the impedance match improves and the radiation efficiency increases, but emissions are undesirable. On the other side, the efficiency decrement of the EBG patch attenuates these frequencies when the higher-order modes occur.

Fig. 5 shows the suppression factor as the ratio of the two different efficiencies, highlighting how the EBG based solution provides an improvement of over $1 \mathrm{~dB}$ within the $2.4 \mathrm{GHz}$ ISM band (marked in blue), while it rejects frequencies above $f_{\mathrm{c}}=3.05 \mathrm{GHz}$ from a minimum of 1 up to over $10 \mathrm{~dB}$. The higher-order resonances of the structures makes it radiating 


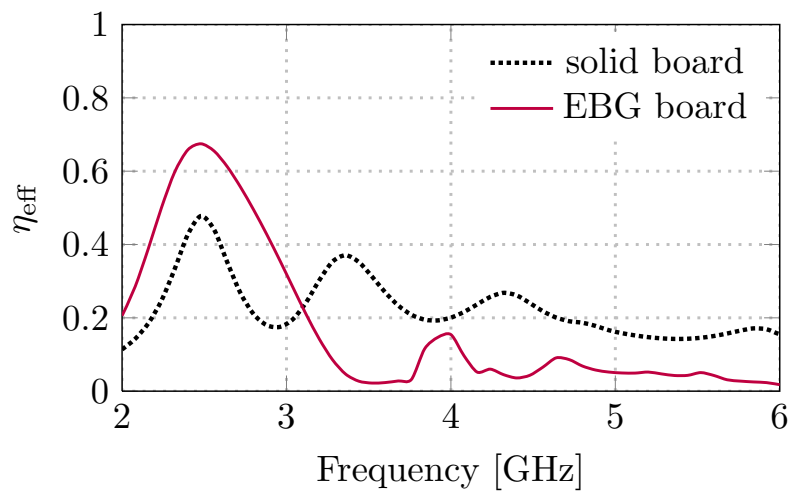

Fig. 4: Efficiency versus frequency for the two antennas: solid ground (solid line) and EBG ground (dashed line).

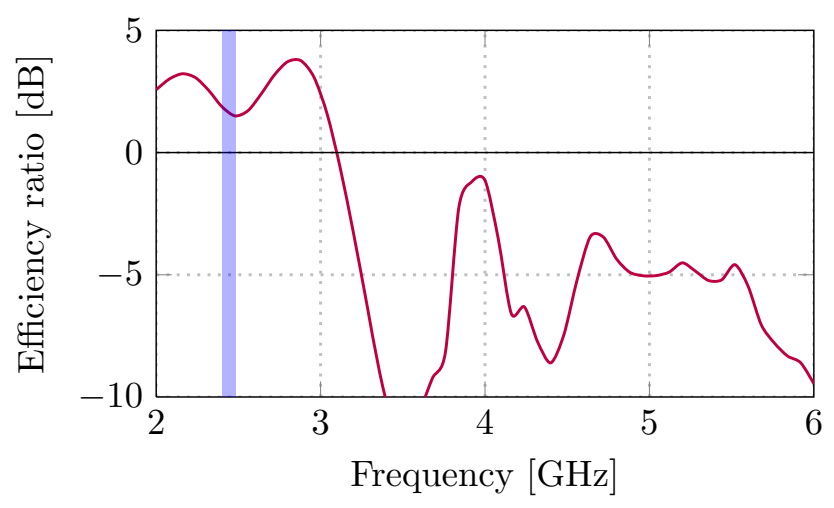

Fig. 5: Another representation of the maximum rejection achievable, with the ratio between the EBG plane over the solid plane expressed in $\mathrm{dB}$. The $2.4 \mathrm{GHz}$ ISM band is highlighted in blue.

far beyond the required frequency range. On the contrary, the EBG plane effectively inhibits such mechanism.

This apparently contradicts the praxis, that wants solid ground planes to be one of the most robust solution to decrease the risk of EMC issues from PCB boards. Instead, in cases in which direct coupling to the antenna feed or parallel plate noise can arise, an EBG etched layer can improve such performance attenuating the higher-order resonances. Nevertheless, we shall not forget that in an integrated product, the same ground plane is shared by other functions, as can be ICs or audio components, so that further investigation with focus on signal integrity performance is necessary before prematurely drawing the above-mentioned conclusion.

\section{CONCLUSION}

A patch antenna implemented on an EBG ground plane was investigated in this paper, and its advantages for product integration examined. In particular, it was noted that such an antenna may obtain higher efficiency while dimensions are shrunk as compared to a traditional design. The advantage is strengthen by the fact that the antenna is more frequency selective, i.e., it attenuates frequencies above a certain frequency $f_{c}$. This helps both into attenuating higher order harmonics and other unwanted radiation of an amount from a few $\mathrm{dB}$ up to 10 and above. Implementation in compact electronic products is advised, when complemented by a careful signal integrity analysis.

\section{REFERENCES}

[1] S. H. Kvist, J. Thaysen, and K. B. Jakobsen, "Improvement of the Earto-Ear Path Gain At $2.45 \mathrm{GHz}$ Using Parasitic Antenna Element," 6th European Conference on Antennas and Propagation, vol. 2, pp. 944 947, 2011.

[2] F. D. Paulis and L. Raimondo, "Compact Configuration for Common Mode Filter Design based on Planar Electromagnetic Bandgap Structures," IEEE Transactions on Electromagnetic Compatibility, vol. 54, no. 3, pp. 646-654, 2012.

[3] S. Shahparnia and O. Ramahi, "Electromagnetic interference (EMI) reduction from printed circuit boards (PCB) using electromagnetic bandgap structures," IEEE Transactions on Electromagnetic Compatibility, vol. 46, no. 4, pp. 580-587, 2004.

[4] J. Qin, O. Ramahi, and V. Granatstein, "Novel planar electromagnetic bandgap structures for mitigation of switching noise and EMI reduction in high-speed circuits," IEEE Transactions on Electromagnetic Compatibility, vol. 49, no. 3, pp. 661-669, 2007.

[5] M. Kim, K. Koo, C. Hwang, Y. Shim, J. Kim, and J. Kim "A Compact and Wideband Electromagnetic Bandgap Structure Using a Defected Ground Structure for Power/Ground Noise Suppression in Multilayer Packages and PCBs," IEEE Transactions on Electromagnetic Compatibility, vol. 54, no. 3, pp. 689-695, Jun. 2012.

[6] M. M. Bait-Suwailam and O. M. Ramahi, "Ultrawideband Mitigation of Simultaneous Switching Noise and EMI Reduction in High-Speed PCBs Using Complementary Split-Ring Resonators," IEEE Transactions on Electromagnetic Compatibility, vol. 54, no. 2, pp. 389-396, Apr. 2012.

[7] A. Ruaro, J. Thaysen, and K. B. Jakobsen, "Metamaterial-inspired Nearfield Resonant Parasitic Structure for Directional Suppression of Narrowband EMI/RFI in Compact Systems," in 2013 National Radio Science Meeting (NRSM) (Joint with AP-S Symposium), 2013.

[8] _ , "Mitigation of Unwanted Forward Narrow-band Radiation from PCBs with a Metamaterial Unit Cell," in 2013 European Microwave Conference (EuMC), 2013.

[9] J. Liang and H. D. Yang, "Radiation Characteristics of a Microstrip Patch Over an Electromagnetic Bandgap Surface," IEEE Transactions on Antennas and Propagation, vol. 55, no. 6, pp. 1691-1697, 2007.

[10] H. Mosallaei and K. Sarabandi, "Antenna Miniaturization and Bandwidth Enhancement Using a Reactive Impedance Substrate," IEEE Transactions on Antennas and Propagation, vol. 52, no. 9, pp. 24032414, 2004.

[11] K. H. Kim and J. E. Schutt-ainé, "Design of EBG Power Distribution Networks With VHF-Band Cutoff Frequency and Small Unit Cell Size for Mixed-Signal Systems," IEEE Microwave and Wireless Components Letters, vol. 17, no. 7, pp. 489-491, 2007.

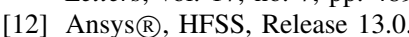

